\title{
A single-institution review of image-guided brachytherapy for vaginal malignancies using customized molded applicators and interstitial needles
}

\author{
Emily Flower, MAppSci,2, Salman Zanjani, BAppScil, Gemma Busuttil, BAppScil, Emma Sullivan, MScl, \\ Wayne Smith, BAppScil, Kathy Tran, BAppScil, David Thwaites, MA, MSc, PhD, FIPEM FlnstP FRCR (hon) FACPSEM!', \\ Jennifer Chard, MBBSl, Viet Do, MBBS, MPH, MHM, PhD 3.4 \\ 'Department of Radiation Oncology, Sydney West Radiation Oncology Network, Crown Princess Mary Cancer Centre, Westmead, Australia, \\ ${ }^{2}$ Institute of Medical Physics, School of Physics, University of Sydney, Australia, ${ }^{3}$ Radiation Oncology, Liverpool CTC, Liverpool Hospital, \\ Sydney, Australia, ${ }^{4}$ South Western Sydney Clinical School, UNSW, Australia
}

Research conducted at: Crown Princess Mary Cancer Centre, Westmead Hospital, Westmead, NSW 2145 Australia

\begin{abstract}
Purpose: To review a single-institution's pattern of practice, dosimetry results, and clinical outcomes for patients with unresectable malignancies of vagina, vulva, or urethra, receiving brachytherapy using customized vaginal moulds with or without interstitial needles.

Material and methods: Twenty-one consecutive patients were reviewed. Patients were treated using customized moulds with or without interstitial needles, implanted with a free-hand technique. Technical implementation, such as type of implant and imaging used were recorded. $\mathrm{D}_{90}$ and $\mathrm{D}_{98}$ of clinical target volume $(\mathrm{CTV}), \mathrm{D}_{0.1 \mathrm{cc}}$ of urethra, and $\mathrm{D}_{2 \mathrm{cc}}$ and $\mathrm{D}_{0.1 \mathrm{cc}}$ of bladder and rectum were analyzed. Any adverse clinical outcomes were reported.

Results: Eleven patients experienced endometrial cancer recurrences, one a cervical cancer recurrence and nine vaginal or peri-urethral primary disease. After a median follow-up of 3.5 years, local control was achieved in 14 patients $(67 \%)$. Median $\mathrm{D}_{98}$ and $\mathrm{D}_{90}$ to CTV was $73.7 \mathrm{~Gy}$ and $78.3 \mathrm{~Gy}$, respectively. One patient died from disease progression, one developed distant metastasis, and seven failed locally. Median $\mathrm{D}_{2 \mathrm{cc}}$ to bladder was $64.8 \mathrm{~Gy}$, with low-grade toxicity reported. Median $\mathrm{D}_{2 \mathrm{cc}}$ to rectum was $62.9 \mathrm{~Gy}$, with low-grade toxicity and one case of rectal proctitis was observed. Median urethral $\mathrm{D}_{0.1 \mathrm{cc}}$ was $66 \mathrm{~Gy}$, with no toxicity reported. One patient suffered from a sacral insufficiency fracture. It was presumed that vaginal mucosa proximal to CTV received the total dose, with two patients developing vaginal ulcers, which both resolved; 10 patients reported mild telangiectasia, fibrosis, or stenosis.

Conclusions: A review of patients treated with a customized vaginal mould and interstitial needles showed acceptable doses to CTV, with local control achieved in $67 \%$ of patients, and reasonable doses to organs at risk (OARs) and acceptable toxicity.

J Contemp Brachytherapy 2021; 13, 6: 663-669 DOI: https://doi.org/10.5114/jcb.2021.110347
\end{abstract}

Key words: vagina, endometrial recurrence, brachytherapy.

\section{Purpose}

Primary and recurrent malignancies of the vagina, vulva, or urethra are rare diseases [1], with approximately 70 women diagnosed per year in Australia [2]. Treatment with surgery is difficult due to proximity of organs at risk. The American Brachytherapy Society have provided guidelines or consensus documents for treating primary vaginal disease [1] and salvage treatment for endometrial recurrences [3]. Brachytherapy (BRT) has been shown to be beneficial for local control for both primary [1, 4-12] and recurrent [5-9, 13-19] disease of the vagina, with acceptable risk of toxicity.

External beam radiotherapy (EBRT) with a brachytherapy boost is recommended due to risk of lymphatic involvement [20], although there are reports of a single-
Address for correspondence: Emily Flower, Crown Princess Mary Cancer Centre, Westmead Hospital, Westmead, NSW 2145 Australia, phone: +61-40-404-03-28, ø e-mail: emily.flower@health.nsw.gov.au
Received: 05.06 .2021 Accepted: 05.08 .2021 Published: 30.12 .2021 
modality being used $[11,15,21,22]$. Higher $\mathrm{EQD}_{2}$ total doses were delivered with external beam radiotherapy, followed by a brachytherapy boost [15]. External beam radiotherapy also reduces the volume of gross target prior to brachytherapy. Patients who received combined EBRT and BRT presented with better overall survival [3, 15]. Also, local control was found to be favorable with combined EBRT and BRT [23, 24]. Brachytherapy (alone or in combination with EBRT) was reported to benefit overall survival compared with EBRT alone [25].

Recommendations from the published literature can be summarized as follows: 1. For disease depths less than $0.5 \mathrm{~cm}$, intracavitary brachytherapy is suitable, and 2. Thicker lesions require interstitial brachytherapy [4]. If an intracavitary approach is used, multiple channels work better than single-channel vaginal cylinders [26]. Intracavitary brachytherapy can be delivered using either single- or multi-channel applicators; however, single-channel applicator usage is limited for vaginal cancers due to inability to effectively shape the dose [27]. Albano et al. [28] described a method for taking a mould of the vagina to manufacture a customized applicator for intracavitary brachytherapy. Interstitial brachytherapy can be delivered using either a free-hand or template technique $[1,15,22]$. Additionally, combined interstitial
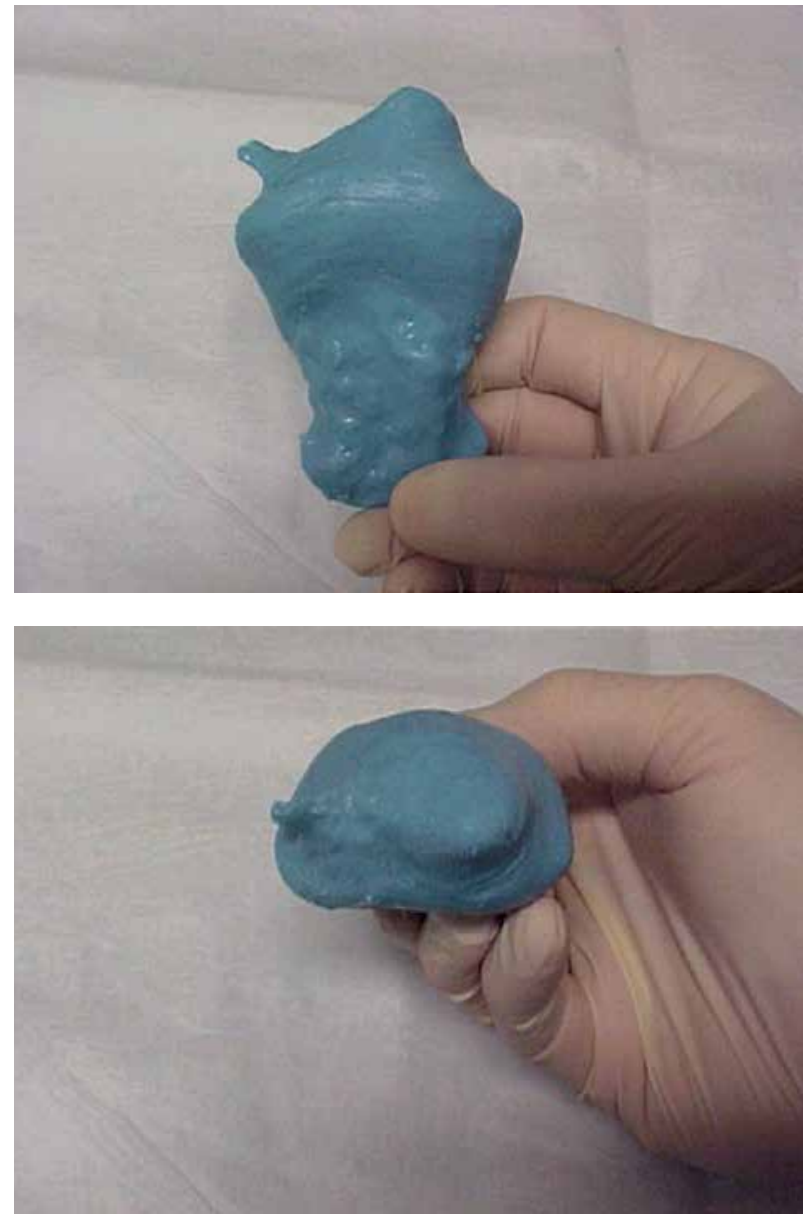
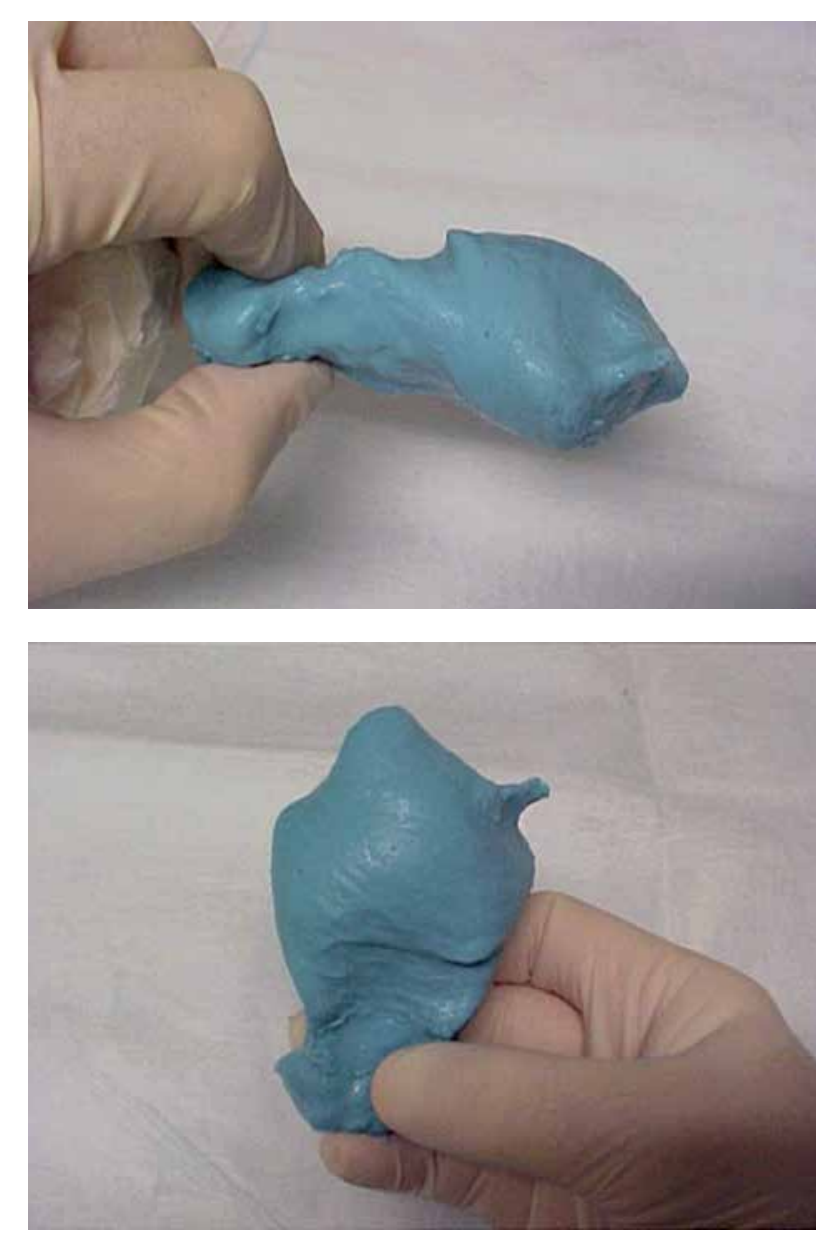

and intracavitary brachytherapy has been used for vaginal cancers.

Dosimetric comparisons of interstitial and multi-channel vaginal applicators have found that interstitial brachytherapy provides lower doses to organs at risk, but for circumferential disease, multi-channel vaginal applicators deliver a better dose to vaginal mucosa [30, 31].

Magnetic resonance imaging (MRI) and 3D planning are recommended for planning vaginal brachytherapy [27]. Organ at risk toxicity for gynecological brachytherapy has been extensively studied in the setting of cervix cancer. Dose-volume histogram parameters, such as $\mathrm{D}_{0.1 \mathrm{cc}}$ $\mathrm{D}_{1 \mathrm{cc}}$ and $\mathrm{D}_{2 \mathrm{cc}}$, have been found to be predictive of toxicity, including correlation with a grade of toxicity [32-37]. Kasibhatla et al. recommended limiting a rectal dose to less than 76 Gy for interstitial brachytherapy for advanced gynecological malignancies [38]. Severe urethral toxicity has been correlated with $D_{0.1 c c}$ [39]. Sigmoid toxicity is usually not a concern for vaginal brachytherapy, due to the distance between recto-sigmoid junction and the vagina. Murakami et al. found that re-irradiation and vaginal $\mathrm{D}_{2 \mathrm{cc}}$ were significant predictors of vaginal ulcers in interstitial brachytherapy for gynecological malignancies [40].

This report presented a single-institution's pattern of practice, dosimetry results, and clinical outcomes for pa-

Fig. 1. Manufacture of acrylic mould 
tients with unresectable malignancies of the vagina, vulva, or urethra, receiving brachytherapy delivery using customized vaginal moulds with or without interstitial needles.

\section{Material and methods}

Following an examination under anesthesia and often an MRI, patients received pelvic external beam radiotherapy. During the initial examination under anesthesia, most of patients received gold seeds to mark the size and location of the original tumor.

External beam radiotherapy was delivered using either a 4-field box conformal technique with $18 \mathrm{MV}$ or volumetric arc radiotherapy, using either $6 \mathrm{MV}$ or $10 \mathrm{MV}$. The prescriptions were 45-50.4 Gy in 25-28 fractions. Two patients (of 21 reviewed) had previous radiation treatment to the pelvis and did not receive external beam radiotherapy prior to their brachytherapy. One of these patients had previously had an unrelated rectal cancer, with external beam radiation ( $50 \mathrm{~Gy} / 25$ fractions, 22 months previously). The other had a vaginal cylinder brachytherapy following primary endometrial cancer (21 Gy/3 fractions, prescribed to a depth of $5 \mathrm{~mm}, 5.5$ years previous$1 y)$, and was then treated again for a recurrence.

Brachytherapy was performed as a boost, following external beam radiotherapy. The patient was anesthe-
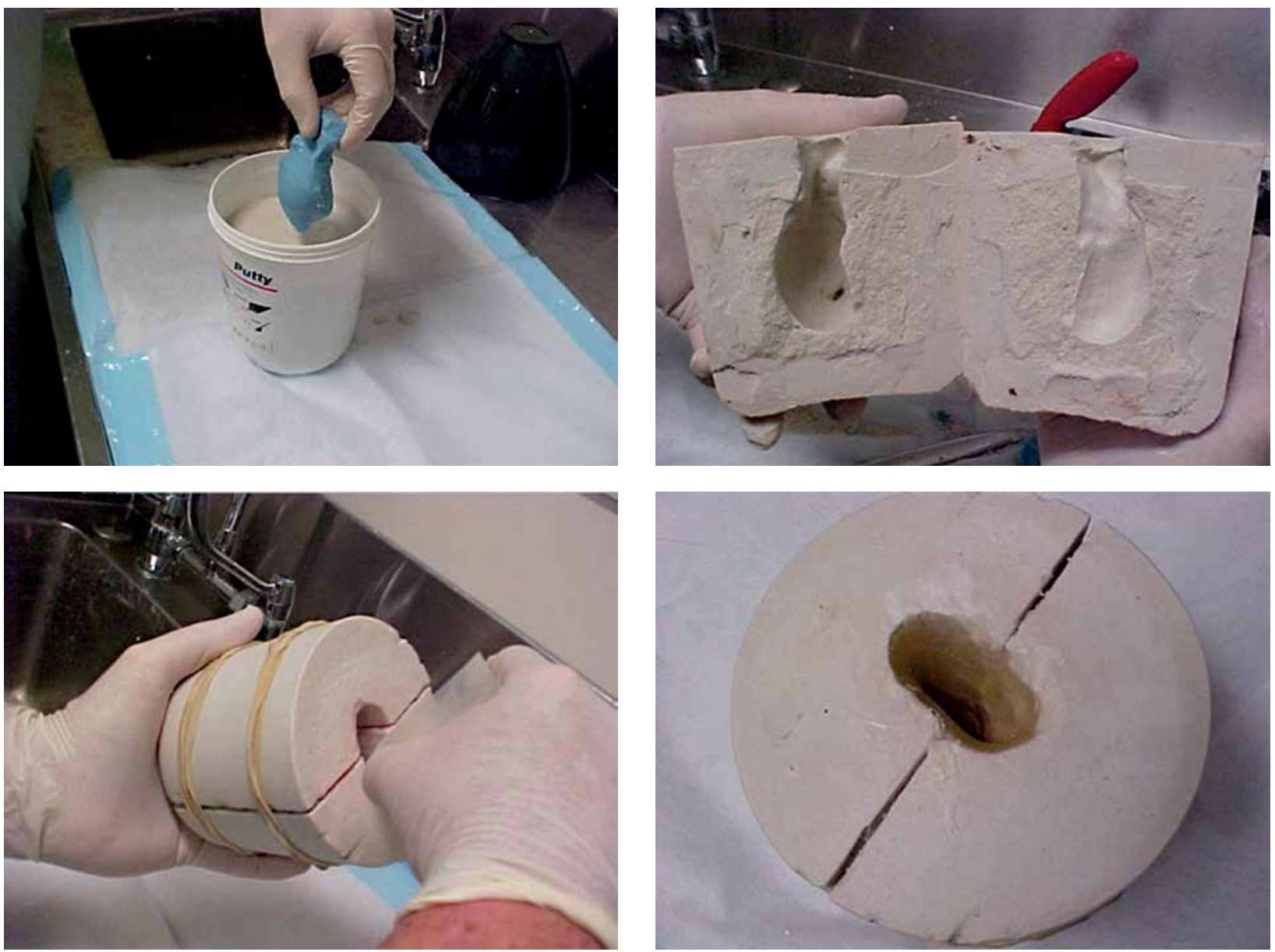

tized, and an examination was performed. A mould for a customized applicator was made using a similar technique, as described by Albano et al. [28]. Dental alginate was applied to form an impression of the vaginal canal (Figure 1). This mould was then placed in quick setting gypsum plaster. Then, the plaster mould was used to create a thin acrylic applicator shell conformal to the vaginal canal. 4.7 French catheters were inserted into the acrylic mould in such a way, as to enable the dose distribution to be conformal to clinical target volume. Wax filling was applied to stabilize the catheters within the mould. Figure 2 shows a mould construction process. Figure 3 presents a completed customized applicator.

Whenever required, interstitial needles were placed along the lateral vaginal wall near the clinical target volume, using a free-hand technique. Interstitial needles were inserted prior to the mould, so the oncologist could palpate the path of needles from within the vaginal canal. The mould was inserted into the patient and surgically stitched into place.

After recovering from anesthetic, the patient had a computed tomography (CT) scan at which, radioopaque CT marker wires were inserted into all channels within the mould and needles. If MRI was required (and not contraindicated), the markers were removed prior to imaging. MRI was not performed if there was a lack of

Fig. 2. Acrylic shell manufacturer 


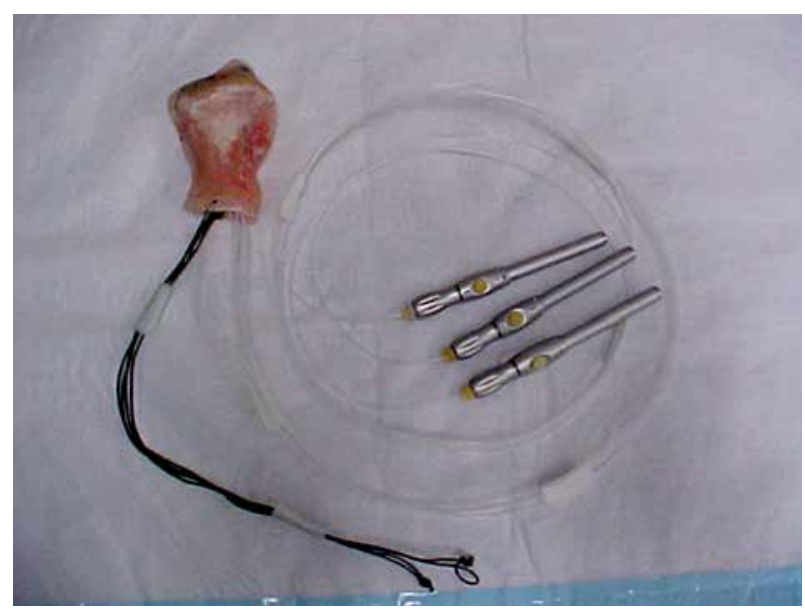

Fig. 3. Completed customized applicator

residual tumor evident during the examination under anesthesia, or if MRI was contraindicated for the patient. T2 fast spin echo sequences were performed with axial, sagittal and coronal planes.

CT and MR images (if applicable) were fused using rigid image registration, matching the mould applicator, needles, and gold seeds. Organs at risk and clinical target volumes were delineated on MRI (if available, otherwise CT), whilst CT was used for an applicator reconstruction.

Using BrachyVision (Varian Medical Systems, Palo Alto, USA), a plan was generated to deliver a prescription dose to clinical target volume (CTV) $\mathrm{D}_{90 \%} \geq 75$ Gy total dose $\mathrm{EQD}_{2}$, while maximizing conformity and limiting the dose to organs at risk, with dose constraints to $D_{0.1 \mathrm{cc}}$ and $\mathrm{D}_{2 \mathrm{cc}}$ of the bladder, rectum, and sigmoid-colon, and $\mathrm{D}_{0.1 \mathrm{cc}}$ to the urethra. Dwell time optimization was performed using a combination of volumetric and/or graphical optimization techniques. Dose formalism described by the American Association of Physicists in Medicine Task Group 43 was applied for dose calculations [29]. Total EQD 2 bladder and rectum planning objectives were $D_{2 c c} \leq 90$ Gy and $D_{2 c c} \leq 75$ Gy, respectively.

After a quality review, the plan was approved by a radiation oncologist, and treatment was delivered using a Varisource iX (Varian Medical Systems, Palo Alto, USA). The patients usually remained in hospital overnight and two additional fractions were given the following day (if four fractions were applied, patient stayed two nights), allowing a minimum of six hours between fractions. A combination of verification measurements, including protruding needle length, verification CT scan,

Table 1. Diagnosis for patients treated with customized mould and/or needles

\begin{tabular}{lc} 
Number of patients & Diagnosis \\
\hline 11 & Endometrial recurrence \\
\hline 7 & Vagina primary \\
\hline 1 & Cervix recurrence \\
\hline 1 & Urethra primary \\
\hline 1 & Vulva primary
\end{tabular}

and fluoroscopic imaging were performed prior to each additional fraction as appropriate for each case.

For this audit, medical records of the patients were accessed to determine treatment response, disease recurrence (local or distant), and toxicity as well as dosimetric data, such as CTV $\mathrm{D}_{90}$ and $\mathrm{D}_{98}$ for bladder and rectum $\mathrm{D}_{2 \mathrm{cc}}$ and $\mathrm{D}_{0.1 \mathrm{cc}}$ and urethral $\mathrm{D}_{0.1 \mathrm{cc}}$. Dose homogeneity within CTV and dose conformity were calculated using dose homogeneity index (Eq. 1) [41] and simplified conformity index (Eq. 2) [42].

$$
\begin{aligned}
& D H I=1-\frac{V_{C T V, 150}}{V_{C T V, 100}} \\
& C I=\frac{V_{C T V, 100}}{V_{C T V}} \times \frac{V_{C T V, 100}}{V_{R E F}}
\end{aligned}
$$

Where DHI was the dose homogeneity index, $\mathrm{V}_{\mathrm{CTV}, 150}$ was the volume of the CTV that receives $150 \%$ of the prescribed dose, $\mathrm{V}_{\mathrm{CTV}, 100}$ was the volume of the CTV that receives $100 \%$ of the prescribed dose, CI was the conformity index, $\mathrm{V}_{\mathrm{CTV}}$ was the volume of the $\mathrm{CTV}$, and $\mathrm{V}_{\text {ref }}$ was the volume of the $100 \%$ isodose.

\section{Results}

Twenty-one consecutively treated patients were reviewed, following a treatment between 2010 and 2018. Table 1 shows details of diagnoses for the patients' cohort of this study. The mean follow-up was 4.0 years (range, 1.6-9.2 years), and the mean age was 70 years (range, $54-88$ years).

Ten patients were treated using only channels within the customized mould. For eleven patients, free-hand interstitial needles were used in addition to the channels within the mould. For one patient, all dwell positions were in interstitial needles, with the customized mould being used only to stabilize the vaginal anatomy. The average number of channels per patient was 5 , with a range of 2-12 (including interstitial needles). When interstitial needles were required, either 2 or 3 needles were inserted.

Six patients were planned using CT imaging only, and the remaining patients had CT and MR imaging for planning. CT enabled catheter reconstruction within the mould.

$\mathrm{EQD}_{2}$ values were calculated assuming an $\alpha / \beta$ ratio of 10 for target volumes and 3 for late responding normal tissue. Sixteen patients were treated over three fractions and five patients received four fractions. The average CTV volume was $14.3 \mathrm{~cm}^{3}$ (range, $1.5 \mathrm{~cm}^{3}$ to $47.3 \mathrm{~cm}^{3}$ ). CTV and organ at risk plan quality metrics (PQM) and toxicity are presented in Table 2 . Figure 4 shows an example of an MR image with the customized applicator, $\mathrm{CTV}$, and isodoses.

Local disease control was achieved for $67 \%$ of the patients. Disease-related adverse outcomes included two incomplete responses, leaving residual vaginal tumor and local recurrences in five patients, and one out of field recurrence in the groin. One of the local progression cases died 28 months after brachytherapy. One patient with a recurrence had a vaginal resection and continues to remain disease-free at 31 months.

It can be assumed that the vaginal mucosa proximal to CTV received the prescribed dose. Two patients developed vaginal ulcers, one responded to hyperbaric oxygen 
Table 2. Plan quality metrics and toxicity

\begin{tabular}{|c|c|c|c|c|}
\hline & Structure/organ & Plan quality metric (PQM) & Median & Toxicity \\
\hline \multirow[t]{4}{*}{ Target } & \multirow[t]{4}{*}{ CTV } & $\mathrm{D}_{98}\left(\mathrm{~Gy}, \mathrm{EQD}_{2}\right)$ & 73.7 & \\
\hline & & $\mathrm{D}_{90}\left(\mathrm{~Gy}, \mathrm{EQD}_{2}\right)$ & 78.3 & \\
\hline & & $\mathrm{DHI}$ & 0.5 & \\
\hline & & $\mathrm{Cl}$ & 0.4 & \\
\hline \multirow[t]{6}{*}{ Organs at risk } & \multirow[t]{2}{*}{ Bladder } & $\mathrm{D}_{2 \mathrm{cc}}\left(\mathrm{Gy}, \mathrm{EQD}_{2}\right)$ & 64.8 & \multirow[t]{2}{*}{ Low-grade toxicity } \\
\hline & & $D_{0.1 c c}\left(G y, E Q D_{2}\right)$ & 81.2 & \\
\hline & Urethra & $\mathrm{D}_{0.1 \mathrm{cc}}\left(\mathrm{Gy}, \mathrm{EQD} \mathrm{D}_{2}\right)$ & 66.0 & None recorded \\
\hline & \multirow[t]{2}{*}{ Rectum } & $\mathrm{D}_{2 \mathrm{cc}}\left(\mathrm{Gy}, \mathrm{EQD}_{2}\right)$ & 62.9 & \multirow{2}{*}{$\begin{array}{l}\text { Low-grade toxicity, one patient developed rectal } \\
\text { proctitis }\end{array}$} \\
\hline & & $\mathrm{D}_{0.1 \mathrm{ccc}}\left(\mathrm{Gy}, \mathrm{EQD} \mathrm{D}_{2}\right)$ & 80.3 & \\
\hline & Sacrum & & & One patient had insufficiency fracture \\
\hline
\end{tabular}

therapy, one resolved with time, and 10 patients reported mild vaginal telangiectasia, fibrosis, or stenosis.

Two of the patients in this series had previous pelvic radiation treatment. One received a prophylactic cylinder brachytherapy treatment of 21 Gy in 3 fractions, and one had previous EBRT for a rectal carcinoma.

\section{Discussion}

Using a combination of customized molded applicators and free-hand interstitial needles, acceptable doses to both the target and organs at risk were achievable. Given the challenging anatomical positions of some of these tumors, combined with limitations of curvature in the source path, there were often very limited dwell positions that could be used to deliver the dose, which affected the conformity of the doses around the small volumes.

Ideally, a direct dosimetric comparison of a multichannel applicator plan using the mould technique would be performed as a planning study to determine which of the two gives the highest quality of the plan. However, this was not achievable with the data available, as the shape of the vagina was different between the two types of insertions.

There are many uncertainties related to brachytherapy for vaginal malignancies. Given the rarity of these tumors, most data comes from small single-institution studies. This leads to a lack of consensus for prescription doses and fractionation as well as contouring uncertainties. A range of practice related to contouring clinical target volumes for recurrent endometrial treatment has been reported, including variation in reporting and prescription to CTVs and variation with different imaging techniques (CT and MRI) [3].

Two patients in this series had a history of previous pelvic radiotherapy. Sadozye et al. [43] reviewed re-irradiation in the setting of recurrent gynecological cancers and concluded there were significant uncertainties regarding treatment modality and doses in this setting. Kamrava et al. [3] suggested that there were greater uncertainties in a re-treatment setting as well as higher risk of toxicity and overall worse outcomes [3]. Yoshida et al. [44] reported higher vaginal mucosa reactions for re-irradiation. A single-institution audit of re-irradiated gy-

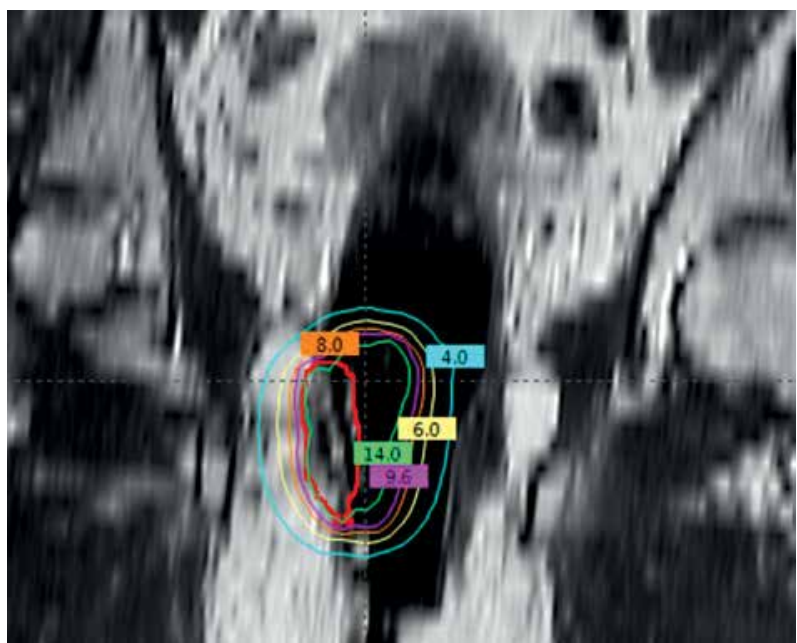

Fig. 4. A frontal view showing an MR image with customized applicator in situ. Clinical target volume (CTV) is displayed with red contour, and isodoses are also shown

necological malignancies found that it gave a reasonable chance of long-term local control and acceptable organ at risk toxicity [14]. Whilst not statistically significant, the two patients in the current series who developed vaginal necrosis were the two who previously received radiotherapy to the pelvis. Ling et al. [45] concluded that if OAR doses were limited, successful salvage could be achieved for $40 \%$ of patients with recurrent vaginal disease, similar to the results reported in this series. Raziee et al. [46] found that re-irradiation provided a safe and effective salvage option for over a quarter of patients.

The most significant prognostic factor was reported to be the FIGO tumor stage, with other prognostic factors being the size and location of tumor [11]. Higher doses have been shown to improve local control for recurrent endometrial cancer [47-50]. 3D image guidance also seems to be assisting in local control outcomes [13, 51, 52].

Chronic side effects from radiotherapy for vaginal cancers are relatively rare. Grade 1-4 side effects have been reported for bladder and rectum at $\leq 2 \%$ and up to $6 \%$ for vaginal side effects [11]. Moreover, dilator use has been shown to decrease vaginal stenosis $[53,54]$. 
There are also different methods reported to produce customized moulds. Nilsson et al. used a two-part putty [55]. However, further work is warranted investigating the use of $3 \mathrm{D}$ printing in the treatment of vaginal malignancies $[56,57]$.

\section{Conclusions}

A review of patients treated with a customized vaginal mould and interstitial needles showed that this technique allows for acceptable doses to CTV with local control achieved in $67 \%$ of the patients as well as reasonable doses to organs at risk and acceptable toxicity.

\section{Disclosure}

The authors report no conflict of interest.

\section{References}

1. Beriwal S, Demanes D, Erickson B et al. American Brachytherapy Society consensus guidelines for interstitial brachytherapy for vaginal cancer. Brachytherapy 2012; 11: 68-75.

2. NSW Cancer Council. https://www.cancercouncil.com.au/ vaginal-cancer

3. Kamrava M, Beriwal S, Erickson B et al. American Brachytherapy Society recurrent carcinoma of the endometrium task force patterns of care and review of the literature. Brachytherapy 2017; 16: 1129-1143.

4. Beriwal S, Rwigema J, Higgins E et al. Three-dimensional image-based high-dose-rate interstitial brachytherapy for vagina cancer. Brachytherapy 2012; 11: 176-180.

5. BeriwalS, Heron D, Mogus R et al. High-dose rate brachytherapy (HDRB) for primary or recurrent cancer in the vagina. Radiat Oncol 2008; 3: 7.

6. Fokdal L, Tanderup K, Neilsen S et al. Image and laparoscopic guided interstitial brachytherapy for locally advanced primary or recurrent gynaecological cancer using the adaptive GEC ESTRO target concept. Radiother Oncol 2011; 100: 473-479.

7. Thibault I, Lavallée M, Aubin S et al. Inverse-planned gynaecological high-dose-rate interstitial brachytherapy: Clinical outcomes and dose-volume histogram analysis. Brachytherapy 2012; 11: 181-191.

8. D'Souza D, Wiebe E, Patil N et al. CT-based interstitial brachytherapy in advanced gynecologic malignancies: Outcomes from a single institution experience. Brachytherapy 2014; 13: 225-232.

9. De Ieso P, Mullassery V, Shrimali R et al. Image-guided vulvovaginal interstitial brachytherapy in the treatment of primary and recurrent gynecological malignancies. Brachytherapy 2012; 11: 306-310.

10. Aldridges $\mathrm{P}$, Onderdonk $\mathrm{B}$, Cunningham $\mathrm{M}$ et al. Institutional experience using interstitial brachytherapy for the treatment of primary and recurrent pelvic malignancies. $J$ Contemp Brachytherapy 2016; 8: 173-180.

11. Mock U, Kucera H, Fellner C et al. High-Dose-Rate (HDR) brachytherapy with or without external beam radiotherapy in the treatment of primary vaginal carcinoma: long-term results and side effects. Int J Rad One Biol Phys 2003; 56: 950-957.

12. Damast S, Takiar V, McCarthy $S$ et al. Treatment of early stage vaginal cancer with EBRT and MRI-based intracavitary brachytherapy: A retrospective case review. Gynecol Oncol Rep 2016; 17: 89-92.

13. Lee L, Dumato A, Viswanathan A. Clinical outcomes following 3D image-guided brachytherapy for vaginal recurrence of endometrial cancer. Gynecol Oncol 2013; 131: 586-592.
14. Zolciak-Siwinska A, Bijok M, Jonska-Gmyrek J et al. HDR brachytherapy for the reirradiation of cervical and vaginal cancer: analysis and dosage delivered to organs at risk. Gynecol Oncol 2014; 132: 93-97.

15. Jhingran A, Burke T, Eifel P. Definitive radiotherapy for patients with isolated vaginal recurrence of endometrial carcinoma after hysterectomy. Int J Rad Oncol Biol Phys 2003; 56: 1366-1372.

16. Hasbini A, Haie-Meder C, Morice P et al. Outcome after salvage radiotherapy (brachytherapy +/- external) in patients with a vaginal recurrence from endometrial carcinomas. Radiother Oncol 2002; 65: 23-28.

17. Chapman C, Maghsoudi K, Littell R et al. Salvage high-doserate brachytherapy and external beam radiotherapy for isolated vaginal recurrences of endometrial cancer with no prior adjuvant therapy. Brachytherapy 2017; 16: 1152-1158.

18. Baek S, Isohashi F, Yamaguchi H et al. Salvage high-doserate brachytherapy for isolated vaginal recurrence of endometrial cancer. Brachytherapy 2016; 15: 812-816.

19. Chopra S, Engineer R, Shah S. MRI and PET guided interstitial brachytherapy for post-surgical vaginal recurrences of cervical cancer: results of phase II study. Int J Radiat Oncol Biol Phys 2020; 106: 310-319.

20. Yeh A, Marcus R, Amdur R et al. Patterns of failure in squamous cell carcinoma of the vagina treated with definitive radiotherapy alone: what is the appropriate treatment volume? Int J Cancer 2001; 96: 109-116.

21. Huh WK, Straughn JM, Mariani A et al. Salvage of isolated vaginal recurrences in women with surgical stage I endometrial cancer: a multiinstitutional experience. Int J Gynecol Cancer 2007; 17: 886-889.

22. Sekii S, Murakami N, Kato T et al. Outcomes of salvage highdose-rate brachytherapy with or without external beam radiotherapy for isolated vaginal recurrence of endometrial cancer. J Contemp Brachytherapy 2017; 9: 209-215.

23. Sears J, Greven K, Hoen H et al. Prognostic factors and treatment outcome for patients with locally recurrent endometrial cancer. Cancer 1994; 74: 1303-1308.

24. Nag S, Martinez-Monge R, Copeland L et al. Perineal template interstitial brachytherapy salvage for recurrent endometrial adenocarcinoma metastatic to the vagina. Gynecol Oncol 1997; 66: 16-19.

25. Orton A, Boothe D, Williams N et al. Brachytherapy improves survival in primary vaginal cancer. Gynecol Oncol 2016; 141: 501-506.

26. Gebhardt BJ, Vargo JA, Kim H et al. Image-based multichannel vaginal cylinder brachytherapy for the definitive treatment of gynecologic malignancies in the vagina. Gynecol Oncol 2018; 150: 293-299.

27. Glaser S, Beriwal S. Brachytherapy for malignancies of the vagina in the 3D era. J Contemp Brachytherapy 2015; 7: 312-318.

28. Albano M, Dumas I, Haie-Meder C. Brachytherapy at the Institut Gustave-Roussy: personalized vaginal mould applicator: technical modification and improvement. Cancer Radiother 2008; 12: 822-826.

29. Rivard M, Coursey B, DeWerd L et al. Update of AAPM Task Group No. 43 Report: A revised AAPM protocol for brachytherapy dose calculations. Med Phys 2004; 31: 633-674.

30. Kushner DM, Fleming PA, Kennedy AW et al. High dose rate (192)Ir afterloading brachytherapy for cancer of the vagina. Br J Radiol 2003; 76: 719-725.

31. Mendez LC, Paudel M, Wronski M et al. Dosimetric comparison of interstitial brachytherapy with multi-channel vaginal cylinder plans in patients with vaginal tumors. Radiat Oncol 2017; 12: 84.

32. Kim Y, Kim YJ, Kim JY et al. Toxicities and dose-volume histogram parameters of MRI-based brachytherapy for cervical cancer. Brachytherapy 2017; 16: 116-125. 
33. Georg P, Lang S, Dimopoulos JC et al. Dose-volume histogram parameters and late side effects in magnetic resonance image-guided adaptive cervical cancer brachytherapy. Int I Radiat Oncol Biol Phys 2011; 79: 356-362.

34. Georg P, Pötter R, Georg D et al. Dose effect relationship for late side effects of the rectum and urinary bladder in magnetic resonance image-guided adaptive cervix cancer brachytherapy. Int J Radiat Oncol Biol Phys 2012; 82: 653-657.

35. Mazeron R, Maroun P, Castelnau-Marchand P et al. Pulseddose rate image-guided adaptive brachytherapy in cervical cancer: Dose-volume effect relationships for the rectum and bladder. Radiother Oncol 2015; 116: 226-232.

36. Mazeron R, Fokdal LU, Kirchheiner K et al. Dose-volume effect relationships for late rectal morbidity in patients treated with chemoradiation and MRI-guided adaptive brachytherapy for locally advanced cervical cancer: Results from the prospective multicenter EMBRACE study. Radiother Oncol 2016; 120: 412-419.

37. Ribeiro I, Janssen H, De Brabandere M et al. Long term experience with 3D image guided brachytherapy and clinical outcome in cervical cancer patients. Radiother Oncol 2016; 120: 447-454.

38. Kasibhatla M, Clough R, Montana G et al. Predictors of severe gastrointestinal toxicity after external beam radiotherapy and interstitial brachytherapy for advanced or recurrent gynecologic malignancies. Int J Radiat Oncol Biol Phys 2006; 65: 398-403

39. Amsbaugh M, Bhatt N, Hunter T et al. Computed tomography - planned interstitial brachytherapy for locally advanced gynecological cancer: Outcomes and dosimetric predictors of urinary toxicity. Brachytherapy 2016; 15: 49-56.

40. Murakami N, Kasamatsu T, Sumi M et al. Vaginal tolerance of CT based image-guided high-dose rate interstitial brachytherapy for gynecological malignancies. Radiat Oncol 2014; 9: 31

41. Wu A, Ulin K, Sternick ES. A dose homogeneity index for evaluating 192Ir interstitial breast implants. Med Phys 1998; 15: 104-107.

42. Baltas D, Kolotas C, Geramani K et al. A conformal index (COIN) to evaluate implant quality and dose specification in brachytherapy. Int J Radiat Oncol Biol Phys 1998; 40: 515-524.

43. Sadozye AH. Re-irradiation in gynaecological malignancies: a review. Clin Oncol 2018; 30: 110-115.

44. Yoshida K, Yamazaki H, Nakamura S et al. Re-irradiation using interstitial brachytherapy increases vaginal mucosal reaction compared to initial brachytherapy in patients with gynecological cancer. Anticancer Res 2013; 33: 5687-5692.

45. Ling D, Vargo J, Glaser S et al. Outcomes after definitive re-irradiation with $3 \mathrm{D}$ brachytherapy with or without external beam radiation therapy for vaginal recurrence of endometrial cancer. Gynecol Oncol 2019; 152: 581-558.

46. Raziee H, D'Souza D, Velker V et al. Salvage re-irradiation with single-modality interstitial brachytherapy for the treatment of recurrent gynaecological tumours in the pelvis: a multi-institutional study. Clin Oncol 2020; 32: 43-51.

47. Curran W, Whittington R, Peters A et al. Vaginal recurrences of endometrial carcinoma: the prognostic value of staging by a primary vaginal carcinoma system. Int J Radiat Oncol 1988; 15: 803-808.

48. Kuten A, Grigsby P, Perez C et al. Results of radiotherapy in recurrent endometrial carcinoma: a retrospective analysis of 51 patients. Int J Radiat Oncol Biol Phys 1989; 17: 29-34.

49. Morgan J, Reddy S, Sarin P et al. Isolated vaginal recurrences of endometrial carcinoma. Radiology 1993; 189: 609-613.

50. Wylie J, Irwin C, Pintille $\mathrm{M}$ et al. Results of radical radiotherapy for recurrent endometrial cancer. Gynecol Oncol 2000; 77 66-72.
51. Vargo J, Kim H, Houser C et al. Definitive salvage for vaginal recurrence of endometrial cancer: the impact of modern intensity-modulated-radiotherapy with image-based HDR brachytherapy and the interplay of the PORTEC 1 risk stratification. Radiother Oncol 2014; 113: 126-131.

52. Fokdal L, Ørtoft G, Hansen ES et al. Toward four-dimensional image-guided adaptive brachytherapy in locally recurrent endometrial cancer. Brachytherapy 2014; 13: 554-561.

53. Cerentini TM, Schlöttgen J, Viana da Rosa P et al. Clinical and psychological outcomes of the use of vaginal dilators after gynaecological brachytherapy: a randomized clinical trial. Adv Ther 2019; 36: 1936-1949.

54. Hanlon A, Small W, Strauss J et al. Dilator use after vaginal brachytherapy for endometrial cancer: a randomized feasibility and adherence study. Cancer Nurs 2018; 41: 200-209.

55. Nilsson S, Moutrie Z, Cheuk R et al. A unique approach to high-dose-rate vaginal mold brachytherapy of gynecologic malignancies. Brachytherapy 2015; 14: 267-272.

56. Laan R, Nout R, Dankelman J et al. MRI-driven design of customised 3D printed gynaecological brachytherapy applicators with curved needle channels. 3D Print Med 2019; 16: 8.

57. Logar HBZ, Hudej R, Šegedin B. Development and assessment of 3D-printed individual applicators in gynecological MRI-guided brachytherapy. J Contemp Brachytherapy 2019; 11: 128-136. 